\title{
HOBt·DCHA-Mediated Synthesis of Sterically Hindered Peptides employing Fmoc-Amino Acid Chlorides in Both Solution-Phase and Solid Phase Methods
}

\author{
Vommina V. Sureshbabu, Naremaddepalli S. Sudarshan \& G. Chenna \\ Krishna
}

To cite this article: Vommina V. Sureshbabu, Naremaddepalli S. Sudarshan \& G. Chenna Krishna (2008) HOBt.DCHA-Mediated Synthesis of Sterically Hindered Peptides employing Fmoc-Amino Acid Chlorides in Both Solution-Phase and Solid Phase Methods, Synthetic Communications, 38:15, 2625-2637, DOI: 10.1080/00397910802219536

To link to this article: http://dx.doi.org/10.1080/00397910802219536

Published online: 09 Sep 2008.

Submit your article to this journal ¿

Џ Article views: 128

View related articles $\sqsubset$

Citing articles: 1 View citing articles $ऍ$ 


\title{
HOBt-DCHA-Mediated Synthesis of Sterically Hindered Peptides employing Fmoc-Amino Acid Chlorides in Both Solution-Phase and Solid Phase Methods
}

\author{
Vommina V. Sureshbabu, Naremaddepalli S. Sudarshan, \\ and G. Chenna Krishna \\ Department of Studies in Chemistry, Central College Campus, \\ Bangalore University, Bangalore, India
}

\begin{abstract}
The synthesis of peptides employing Fmoc-amino acid chlorides in presence of HOBt.DCHA salt in solution as well as by the solid-phase methods is described. The coupling was found to be complete in $30 \mathrm{~min}$ and free from racemization. The synthesis of $\beta$-casomorphin by solid-phase protocol employing Fmoc-amino acid chloride/HOBt.DCHA in $\mathrm{DMF}-\mathrm{CH}_{2} \mathrm{Cl}_{2}$ has also been outlined. The final peptide was obtained in $80 \%$ yield and was fully characterized.
\end{abstract}

Keywords: Fmoc-amino acid chloride, fmoc-amino acid-OBt ester, HOBt-DCHA

\section{INTRODUCTION}

One of the many contributions of Louis Carpino, University of Massachusetts, is the discovery of shelf-stable Fmoc-amino acid chlorides, ${ }^{[1,2]}$ which have been demonstrated to be rapid, efficient, acylating agents useful in peptide chemistry. Because of the high degree of activation associated with them, the acylation using acid chlorides ${ }^{[3,4]}$ makes coupling of very hindered or weakly nucleophlic systems possible, whereas the use of other methods gives inferior results. Those Fmoc-amino acid chlorides bearing a tert-butyl type side chain functional group such as $\mathrm{Asp}(\mathrm{tBu})$,

Received March 18, 2007

Address correspondence to Vommina V. Sureshbabu, Department of Studies in Chemistry, Central College Campus, Bangalore University, Dr. B. R. Ambedkar Veedhi, Bangalore 560 001, India. E-mail:hariccb@rediffmail.com 
Cys(tBu), Lys(Boc), Ser(tBu), $\operatorname{Thr}(\mathrm{tBu}), \quad \operatorname{Trp}(\mathrm{Boc}), \quad \operatorname{Tyr}(\mathrm{tBu})$ and Asp(Pmc) can also be generated using in situ activation via bis(trichlorophenyl)carbonate. The acid chloride coupling mediated by an organic tertiary amine (pyridine, diisopropylethylamine (DIEA)), an essential component of such a reaction protocol, is known to result in the formation of oxazol-5(4H)-one, ${ }^{[5]}$ leading to stereomutation and/or premature deblocking of Fmoc-group, due to the prolongation of the course of the reaction (usually required for the incorporation of hindered amino acids). This has been circumvented by the use of co-coupling agents such as silver cyanide ${ }^{[6,7]}$ potassium salts of 1-hydroxybenzotriazole $(\mathrm{KOBt})^{[8,9]}$ and 1-hydroxy-7-aza-benzotriazole (KOAt) ${ }^{[10,11]}$ t-butyldimethylsilyloxy benzotriazole (TBDMS) derivatives of HOBt (TBMS-OBt) and HOAt (TBDMS-OAt), ${ }^{[12,13]}$ and zinc dust. ${ }^{[14,15]}$ The use of $\mathrm{KOBt} / \mathrm{KOAt} / \mathrm{TBDMS}-\mathrm{OBt}$ or TBDMS-OAt converts acid chloride to its benzotriazole/azabenzotriazole ester, which acts as an acylating agent, whereas in the presence of zinc dust-mediated coupling, the liberated $\mathrm{HCl}$ is directly abstracted. There are few limitations in the use of some of these reagents. The formation of $\mathrm{KCl}$ as a side product avoids the use of KOBt or KOAt in solid phase. The zinc dust-mediated coupling is limited to acylation reactions in solution phase only. TBDMS derivatives cannot be prepared and stored for long periods and need to be freshly prepared every time. Although the use of these co-coupling agents is satisfactory in solution phase, their utility in solid phase is not a straightforward protocol.

This article demonstrates the efficient synthesis of peptides employing Fmoc-amino acid chlorides mediated by HOBt-DCHA salt. Employing the present protocol, the synthesis of $\mathrm{H}_{2} \mathrm{~N}$-Val-Pro-GlyVal-Gly-OH $(\mathrm{VPGVG})^{[16]}$ in solution phase and $\beta$-casomorphin $(\mathrm{H}-\mathrm{T}$ yr-Pro-Phe-Pro-Gly-OH) in solid phase have been accomplished. 1-Hydroxybenzotriazole (HOBt), introduced by Konig and Geiger, ${ }^{[17]}$ is well known to suppress racemization in carbodiimide-mediated couplings. Further, the other popular coupling agents 1-[bis(dimethylamino)methylene]-1H-benzotriazolium hexaflur ophosphate-3-oxide (HBTU) ${ }^{[18]}$ and 2-(1H-benzotriazole-1-yl)1,1,3,3-tetramethylluroniumtetrafluoroborate (TBTU) ${ }^{[19]}$ also possess HOBt as part of their structure. Our interest in developing a simple non-Schotten-Baumann ${ }^{[20]}$ protocol for acid chloride coupling has led to explore the use of HOBt.DCHA salt as a co-coupling agent in base-free conditions. Katritzky et al. have employed the benzotriazole amide derivatives of $\mathrm{N}$-protected amino acids for the acylation of amino acids in an aqueous medium in the presence of triethyl amine ${ }^{[21]}$ In contrast to this, the present synthesis uses the rapidly and in situ generated -OBt ester as acylating agent in a completely base-free organic medium. The Bt-amide derivatives of amino acids are prepared 
through a two-step protocol comprising activation of benzotriazoles with thionyl chloride followed by treatment of $\mathrm{N}$-protected amino acids with activated Bt. However, the Fmoc-acid chlorides are prepared easily in a single step by treating the Fmoc-amino acids directly with thionyl chloride. Also, preparation of the HOBT-DCHA reagent is simple, involving the addition of an equimolar quantity of dicyclohexylamine to anhydrous HOBt in tetrahydrofuran (THF) solution. Upon standing, the salt separates out, which can be crystallized and stored for a long time.

\section{RESULTS AND DISCUSSION}

In a typical reaction, the mixture of Fmoc-amino acid chloride (1 equivalent) and HOBt.DCHA (2.2 equivalents) in THF was initially stirred for about $5 \mathrm{~min}$ to generate-OBt ester (which is evidenced from IR absorption spectrum by the disappearance of the band at $1786 \mathrm{~cm}^{-1}$ pertaining to acid chloride and the appearance of the band at $1820 \mathrm{~cm}^{-1}$ pertaining to $-\mathrm{OBt}$ ester formation), and then amino acid methyl ester salt was added and stirred. As monitored by thin layer chromatography (TLC), high pressure liquid chromatography (HPLC), and infrared spectrum (IR) analysis (Scheme 1), the coupling was found to be complete in about $30 \mathrm{~min}$.

The reaction mixture was filtered to remove the precipitated DCHA. $\mathrm{HCl}$ salt, and after simple workup, the peptide was isolated. The efficacy of HOBt-DCHA-mediated coupling is further demonstrated by the synthesis of dipeptides containing $\mathrm{C}^{\alpha \alpha}$-dialkylglycines ${ }^{[22,23]}\left(\mathrm{H}_{2} \mathrm{~N}\right.$ $\mathrm{CR}^{1} \mathrm{R}^{2}-\mathrm{COOH}$ ) with linear substitutents $[\alpha$-aminoisobutric acid (Aib), diethylglycine (Deg), dipropylglycine (Dpg), and dibutylglycine (Dbg)] and cyclic substitutents [1-aminocyclopentane-1-carboxylic acid $\left(\mathrm{Ac}_{5} \mathrm{c}\right)$, and 1-aminocyclo hexane-1-carboxylic acid $\left(\mathrm{Ac}_{6} \mathrm{c}\right)$, and 1-aminocycloheptane-1-carboxylic acid $\left(\mathrm{Ac}_{7} \mathrm{c}\right)$ ]. The duration of such couplings required about $2 \mathrm{~h}$, and the resulting peptides were obtained in $65-72 \%$ yield (Table 1). Further, the coupling of Fmoc-amino acid-chloride mediated using HOBt.DCHA, and using an aqueous $\mathrm{NaHCO}_{3}$ solution of amino acid, resulted in Fmoc-peptide acid with moderate yield. On the other hand, the use of bis-N,O-trimethylsilyl amino acid ${ }^{[24]}$ gave good yields with analytically pure compounds (Scheme 2).

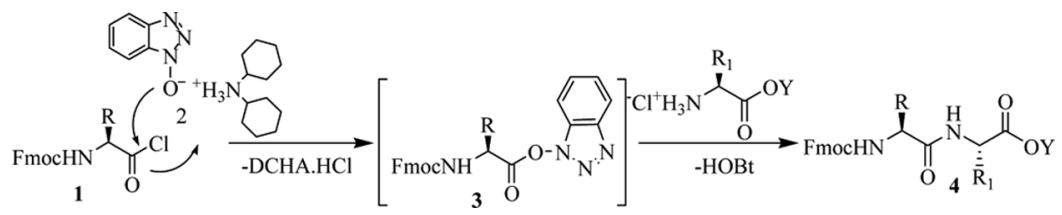

Scheme 1. Synthesis of $N^{\alpha}$-Fmoc-protected peptide esters. 
Table 1. List of peptides made by the HOBt.DCHA method

\begin{tabular}{rlcccc} 
SI. no. & \multicolumn{1}{c}{ Peptide } & Yield $(\%)$ & $\mathrm{Mp}\left({ }^{\circ} \mathrm{C}\right)$ & {$[\alpha]_{\mathrm{D}}{ }^{25}$} & $(c 1.0, \mathrm{DMF})$ \\
\hline 1 & Fmoc-Phg-Phe-OMe & 86 & $194-196$ & +24.0 \\
2 & Fmoc-D-Phg-Phe-OMe & 87 & $194-194$ & -23.4 \\
3 & Fmoc-Leu-Ala-OMe & 88 & $160-162$ & -28.0 \\
4 & Fmoc-Dpg-Dbg-OMe & 72 & gum & - \\
5 & Fmoc-Aib-Aib-OMe & 72 & $70-71$ & - \\
6 & Fmoc-Ac 6 c-Ac 6 c-OMe & 70 & $164-165$ & - \\
7 & Fmoc-Deg-Leu-OMe & 70 & $55-57$ & - \\
8 & Fmoc-Ac Cr-Ac $_{5} \mathrm{c}-\mathrm{OMe}$ & 71 & $207-209$ & - \\
9 & Fmoc-D-Phg-Phe-OH & 82 & $175-177$ & +24.6 \\
10 & Fmoc-Tyr(Bzl)-Pro-OH & 76 & $112-114$ & +38.6 \\
11 & Fmoc-Asp(OBn)-Ala-OH & 80 & $148-149$ & -28.4 \\
12 & Fmoc-Ser(OBn)-Val-OH & 78 & $120-122$ & \\
\hline
\end{tabular}

The ${ }^{1} \mathrm{H}$ NMR analysis of Carpino's diastereomeric dipeptides Fmoc$\mathrm{L}$ or D-Phg-Phe-OMe ${ }^{[2]}$ prepared by employing the present protocol indicated that the C-methylene doublets of phenylalanine and methyl ester singlets are distinct. Thus the coupling is free from racemization. Further, their HPLC analysis [ $\mathrm{R}_{\mathrm{t}}$-values: $\mathrm{L}, \mathrm{L}$-isomer (13.19) and D, L-isomer (14.26)] also confirmed the same. Employing the present protocol for coupling and tris (2-aminoethyl) amine (TAEA) for deprotection of the Fmoc-group, the pentapeptide Fmoc-VPGVG synthesis has been accomplished. The purity of the final peptide Fmoc-VPGVG was satisfactory and was fully characterized by ${ }^{1} \mathrm{H}$ NMR and mass spectra. The yield was $72 \%$.

Although Fmoc-amino acid fluorides ${ }^{[25-27]}$ have been used with or without any organic base successfully as coupling agents in SPPS, the use of Fmoc-amino acid chlorides invariably requires an equimolar quantity of a base. Carpino et al. intially employed a 1:1 mixture of $\mathrm{HOBt}$ and pyridine [or diisopropylethylamine (DIEA) or N-methylmorpholine (NMM)] in the coupling. The addition of a base is well known to promote<smiles>[R]C(NC(F)F)C(=O)On1nnc2ccccc21</smiles>

Scheme 2. Synthesis of $N^{\alpha}$-Fmoc-protected peptide acids. 
one or more side reactions in Fmoc chemistry. The use of already known co-coupling agents (KOBt, KOAt, etc.) is not satisfactory in SPPS.

In the present studies, HOBt.DCHA can be used in SPPS also. This is mainly feasible because of two reasons: 1) HOBt.DCHA dissolves in DMF (or NMP) : $\mathrm{CH}_{2} \mathrm{Cl}_{2}(2: 8)$ and converts Fmoc-amino acid chloride to its-OBt ester, and 2) the side product $\mathrm{DCHA} \cdot \mathrm{HCl}$ salt can be easily and completely removed, after coupling, by washing with a 1:1 mixture of $\mathrm{CHCl}_{3}$ and $\mathrm{CH}_{2} \mathrm{Cl}_{2}$. The solid-phase synthesis of $\beta$-casomorphin using Wang resin as solid support and Fmoc-amino acid chlorides (3 equiv.) and HOBt.DCHA (3 equiv.) in DMF: $\mathrm{CH}_{2} \mathrm{Cl}_{2}$ (2:8) for coupling starting from Fmoc-Gly-O-CH$-\mathrm{C}_{2}-\mathrm{C}_{6} \mathrm{H}_{4}-\mathrm{O}$-resin was carried out. The crude peptide, on HPLC analysis, was found to be about $91 \%$ pure. It was further purified by RP-HPLC and found to be more than $99 \%$ pure. A simple coupling for $30 \mathrm{~min}$ was found to result in the completion of the coupling. Fmoc group was deprotected using $20 \%$ piperidine. After the removal of Fmoc group from Fmoc-Tyr(Bzl)-Pro-Phe-Pro-Gly-OCH${ }_{2}-\mathrm{C}_{6} \mathrm{H}_{4}-\mathrm{O}$ resin, the peptide was cleaved by using $\mathrm{TFA} / \mathrm{H}_{2} \mathrm{O} /$ thioanisole/phenol mixtures at room temperature for $1.5 \mathrm{~h}$ and subjected to catalytic hydrogenation using $10 \% \mathrm{Pd} / \mathrm{C} /$ methanol/hydrogen gas to remove the $\mathrm{Bzl}$ group from the peptide, which was purified.

\section{CONCLUSION}

HOBt.DCHA is a stable and crystalline solid. Its preparation, isolation, and purification is very simple. It has been now demonstrated that the coupling of Fmoc-amino acid chlorides can be accomplished employing HOBt-DCHA under non-Schotten-Baumann conditions. The acylation was efficient, resulting in good yields. The scaling of coupling up to $10 \mathrm{mmol}$ has not posed any practical difficulties. Thus, it has now been demonstrated that HOBt.DCHA finds utility in a solid-phase method for the synthesis of peptides employing Fmoc-amino acid chlorides under non-Schotten-Baumann conditions.

\section{EXPERIMENTAL}

The melting points were determined by the capillary method and are uncorrected. Specific rotations were recorded on a Rudolf Research Autopol IV automatic polarimeter. IR spectra were recorded on a Nicolet model impact 400D FT-IR spectrometer $(\mathrm{KBr}$ pellets, $3 \mathrm{~cm}^{-1}$ resolution) and ${ }^{1} \mathrm{H}$ NMR spectra on a Bruker $400-\mathrm{MHz}$ instrument. Unless otherwise mentioned, all amino acids used have L-configuration. Analytical HPLC was performed on a Waters LC 
3000 system equipped with Waters 484 tunable absorbance UV detector and millipore 745 data module. Water RP 48-deltapack column $(3.9 \mathrm{~mm} \times 300 \mathrm{~mm}, 15 \mu$ spherical) was used for analysis. Thin-layer chromatography (TLC) analysis was carried out using the precoated silica gel $\mathrm{G}_{254}$ plates using 1) $\mathrm{R}_{\mathrm{f}} \mathrm{A} ; \mathrm{CHCl}_{3}$-methanol-acetic acid $40: 2: 1$; 2) $\mathrm{R}_{\mathrm{f}} \mathrm{B}$; ethyl acetate-nhexane $(35: 65)$. Deprotonation of Fmoc group used TAEA, and mass spectra were obtained using a Kratos PCKompact SEQ V1.2.2 spectrometer.

\section{Synthesis of HOBt-DCHA Salt (2)}

Dicyclohexylamine (DCHA) $(0.238 \mathrm{~mL}, 1.2 \mathrm{mmol})$ was added to a suspension of anhydrous $\mathrm{HOBt}(0.135 \mathrm{~g}, 1 \mathrm{mmol})$ in $\mathrm{CH}_{2} \mathrm{Cl}_{2}(5 \mathrm{~mL})$ at $\mathrm{rt}$ and stirred for $1-2 \mathrm{~h}$. Hexane $(2 \mathrm{~mL})$ was added and left for recrystallization. The resulting HOBt.DCHA crystals were filtered, washed with $10 \%$ $\mathrm{CH}_{2} \mathrm{Cl}_{2}$ in hexane $(10 \mathrm{~mL})$, and dried thoroughly to obtain $0.284 \mathrm{~g}(90 \%)$ of the title compound, ${ }^{[28]} \mathrm{mp} 137-139{ }^{\circ} \mathrm{C} ;{ }^{1} \mathrm{H}$ NMR $\left(\mathrm{CDCl}_{3}\right): \delta 1.0-1.7$ $(12 \mathrm{H}, \mathrm{m}), 1.7-2.1(8 \mathrm{H}, \mathrm{m}), 3.05(2 \mathrm{H}, \mathrm{t}), 7.25(2 \mathrm{H}, \mathrm{m}), 7.6-7.8(2 \mathrm{H}$, $\mathrm{m}) ;{ }^{13} \mathrm{C}$ NMR $\left(\mathrm{CDCl}_{3}\right): \delta 24.8,25.0,29.3,52.8,53.0,111.3,118.4,123.4$, 127.7, 143.3; FAB $(\mathrm{M}+1)^{+}: m / z 317.4$.

\section{General Procedure for the Synthesis of $N^{\alpha}$-Protected Peptide Esters (4)}

To a previously stirred solution of Fmoc-amino acid chloride ( $1 \mathrm{mmol})$ and HOBt.DCHA $(0.695 \mathrm{~g}, 2.2 \mathrm{mmol})$ in dry THF $(5 \mathrm{~mL})$, a suspension of amino acid ester hydrochloride salt (1 mmol) in THF $(5 \mathrm{~mL})$ was added, and the reaction mixture was stirred. The completion of the reaction was monitored by TLC. The reaction mixture was filtered and washed with organic solvent (THF, $10 \mathrm{~mL}$ ) to remove $\mathrm{DCHA} \cdot \mathrm{HCl}$ as a white precipitate. The filtrate was concentrated, and $\mathrm{CH}_{2} \mathrm{Cl}_{2}(15 \mathrm{~mL})$ was added. It was washed with $20 \%$ sodium bisulphate $(10 \mathrm{~mL} \times 3)$, to remove excess DCHA, water $(10 \mathrm{~mL} \times 2), 5 \% \mathrm{HCl}(10 \mathrm{~mL} \times 3)$, and water, then dried over anhydrous $\mathrm{Na}_{2} \mathrm{SO}_{4}$. The solvent was removed by evaporation under reduced pressure and product was crystallized using $\mathrm{CH}_{2} \mathrm{Cl}_{2}$-hexane (2:8) to yield the peptide.

\section{Data}

Fmoc-Phg-Phe-OMe: ${ }^{1} \mathrm{H}$ NMR $\left(\mathrm{CDCl}_{3}\right)$ : $\delta 2.91(\mathrm{~d}, 2 \mathrm{H}), 3.10(\mathrm{t}, 1 \mathrm{H}), 3.62$ $(\mathrm{s}, 3 \mathrm{H}), 4.21(\mathrm{t}, 1 \mathrm{H}), 4.53(\mathrm{~d}, 2 \mathrm{H}), 5.20(\mathrm{~d}, 1 \mathrm{H}), 6.23(\mathrm{~d}, 1 \mathrm{H}), 6.91(1 \mathrm{H}, \mathrm{d})$, 
7.30-7.90 (m, 18H); ${ }^{13} \mathrm{C} \mathrm{NMR}\left(\mathrm{CDCl}_{3}\right): \delta 38.2,47.3,52.5,54.4,66.8,67.8$, $120.1,124.1,126.3,126.5,127.2,127.5,128.2,128.7,130.9,133.4,135.8$, 141.5, 144.0, 155.6, 167.5, 171.9; ES MS: $m / z 535.3[\mathrm{M}+\mathrm{H}]^{+}$, Anal. calcd. for $\mathrm{C}_{33} \mathrm{H}_{30} \mathrm{~N}_{2} \mathrm{O}_{5}$ : C, 74.14; H, 5.66; N, 5.24. Found: C, 74.04; H, $5.61 ; \mathrm{N}, 5.28 \%$.

Fmoc-(D)Phg-Phe-OMe: ${ }^{1} \mathrm{H}$ NMR $\left(\mathrm{CDCl}_{3}\right): \delta 2.81(\mathrm{~d}, 2 \mathrm{H}), 3.10(\mathrm{t}$, $1 \mathrm{H}), 3.62(\mathrm{~s}, 3 \mathrm{H}), 4.20(\mathrm{t}, 1 \mathrm{H}), 4.51(\mathrm{~d}, 2 \mathrm{H}), 5.21(\mathrm{~d}, 1 \mathrm{H}), 6.22(\mathrm{~d}, 1 \mathrm{H})$, $6.90(\mathrm{~d}, 1 \mathrm{H}), 7.28-7.90(\mathrm{~m}, 18 \mathrm{H}) ;{ }^{13} \mathrm{C} \mathrm{NMR}\left(\mathrm{CDCl}_{3}\right): 838.3,47.3,52.3$, $54.4,66.8,67.8,120.0,124.0,126.2,126.5,127.2,127.5,128.2,128.7$, 130.9, 133.4, 135.8, 141.5, 144.0, 155.6, 167.5, 171.9; ES MS: $m / z 535.3$ $[\mathrm{M}+\mathrm{H}]^{+}$, Anal. calcd. for $\mathrm{C}_{33} \mathrm{H}_{30} \mathrm{~N}_{2} \mathrm{O}_{5}: \mathrm{C}, 74.14 ; \mathrm{H}, 5.66 ; \mathrm{N}, 5.24$. Found: C, 74;02; H, 5.60; N, 5.30\%.

Fmoc-Leu-Ala-OMe: ${ }^{1} \mathrm{H}$ NMR $\left(\mathrm{CDCl}_{3}\right): \delta 0.97(\mathrm{~d}, 6 \mathrm{H}), 1.62(\mathrm{~m}$, $3 \mathrm{H}), 3.75(\mathrm{~s}, 3 \mathrm{H}), 4.25(\mathrm{t}, 1 \mathrm{H}), 4.41(\mathrm{t}, 1 \mathrm{H}), 4.53(\mathrm{~d}, 2 \mathrm{H}), 5.20(\mathrm{~m}, 1 \mathrm{H})$, $6.33(\mathrm{~m}, 1 \mathrm{H}), 7.27-7.80(\mathrm{~m}, 8 \mathrm{H}) ;{ }^{13} \mathrm{C} \mathrm{NMR}\left(\mathrm{CDCl}_{3}\right): \delta 17.4,22.4,22.9$, $38.3,46.5,47.5,52.3,52.9,66.8,120.0,125.1,127.1,141.5,144.0$, 155.6, 172.0, 173.1; ES MS: $m / z$ 439.4 $[\mathrm{M}+\mathrm{H}]^{+}$, Anal. calcd. for $\mathrm{C}_{25} \mathrm{H}_{30} \mathrm{~N}_{2} \mathrm{O}_{5}$ : C, 68.47; H, 6.90; N, 6.39. Found; C, 68.41; H, 6.70; N, $6.21 \%$.

Fmoc-Dpg-Dbg-Ome: ${ }^{1} \mathrm{H}$ NMR $\left(\mathrm{CDCl}_{3}\right): \delta$ 0.81-2.02 (m, 40H), 3.60 $(\mathrm{s}, 3 \mathrm{H}), 4.11(\mathrm{~d}, 1 \mathrm{H}), 4.20(\mathrm{t}, 2 \mathrm{H}), 6.51(\mathrm{~s}, 1 \mathrm{H}), 6.92(\mathrm{~s}, 1 \mathrm{H}), 7.20-7.81(\mathrm{~m}$, $8 \mathrm{H}) ;{ }^{[13]} \mathrm{C} \mathrm{NMR}\left(\mathrm{CDCl}_{3}\right): \delta 13.3,14.0,18.9,22.3,24.7,37.6,40.9,47.4$, $52.3,61.2,66.7,66.9,120.1,124.0,127.1,127.2,141.4,144.0,155.3$, 170.2, 175.1; ES MS: $m / z 565.6[\mathrm{M}+\mathrm{H}]^{+}$, Anal. Calcd. for $\mathrm{C}_{34} \mathrm{H}_{48} \mathrm{~N}_{2} \mathrm{O}_{5}$ : C, 72.31; H, 8.57; N, 4.96. Found:C, 72.10; H, 8.49; N, $4.90 \%$.

Fmoc-Aib-Aib-Ome: ${ }^{1} \mathrm{H}$ NMR $\left(\mathrm{CDCl}_{3}\right): \delta$ 1.00-1.21 $(\mathrm{m}, 12 \mathrm{H}), 3.80$ $(\mathrm{s}, 3 \mathrm{H}), 4.11(\mathrm{~d}, 1 \mathrm{H}), 4.31(\mathrm{t}, 2 \mathrm{H}), 6.92(\mathrm{~s}, 1 \mathrm{H}), 7.42(\mathrm{~s}, 1 \mathrm{H}), 7.20-7.80$ $(\mathrm{m}, 8 \mathrm{H}) ;{ }^{[13]} \mathrm{C} \mathrm{NMR}\left(\mathrm{CDCl}_{3}\right): \delta 25.2,26.9,47.4,52.1,55.9,60.7,66.9$, 120.0, 124.0, 127.0, 127.6, 141.5, 144.1, 155.3, 171.2, 175.3; ES MS: $m / z 425.5[\mathrm{M}+\mathrm{H}]^{+}$, Anal. calcd. for $\mathrm{C}_{24} \mathrm{H}_{28} \mathrm{~N}_{2} \mathrm{O}_{5}$ : C, 67.91; $\mathrm{H}, 6.65$; N, 6.60. Found: C, 67.90; H, 6.54; N, 6.50\%.

Fmoc-Ac 6 c-Ac $\mathbf{c}_{\mathbf{6}} \mathbf{c}-\mathrm{Ome}:{ }^{1} \mathrm{H}$ NMR $\left(\mathrm{CDCl}_{3}\right): \delta 1.11-2.30(\mathrm{~m}, 20 \mathrm{H})$, $3.71(\mathrm{~s}, 3 \mathrm{H}), 4.20(\mathrm{~d}, 1 \mathrm{H}), 4.52(\mathrm{t}, 2 \mathrm{H}), 6.50(\mathrm{~s}, 1 \mathrm{H}), 7.01(\mathrm{~s}, 1 \mathrm{H}), 7.20$ $7.80(\mathrm{~m}, 8 \mathrm{H}) ;^{[13]} \mathrm{C} \mathrm{NMR}\left(\mathrm{CDCl}_{3}\right): \delta 20.6,24.1,25.5,33.3,38.8,47.5$, 52.3, 60.8, 68.5, 66.8, 120.1, 124.1, 126.3, 127.2, 141.5, 144.7, 155.0, 170.1, 174.5; ES MS: $m / z$ 505.5 $[\mathrm{M}+\mathrm{H}]^{+}$, Anal. calcd. for $\mathrm{C}_{30} \mathrm{H}_{36} \mathrm{~N}_{2} \mathrm{O}_{5}$ : C, 71.41; H, 7.19; N, 5.55. Found: C, 71.01; H, 7.08; N, $5.56 \%$.

Fmoc-Deg-Leu-OMe: ${ }^{1} \mathrm{H}$ NMR $\left(\mathrm{CDCl}_{3}\right): \delta$ 0.81-2.02 $(\mathrm{m}, 18 \mathrm{H}), 3.70$ $(\mathrm{s}, 3 \mathrm{H}), 4.10(\mathrm{~d}, 1 \mathrm{H}), 4.20(\mathrm{~d}, 1 \mathrm{H}), 4.41(\mathrm{t}, 2 \mathrm{H}), 4.60(\mathrm{~d}, 1 \mathrm{H}), 6.81(\mathrm{~s}$, $1 \mathrm{H}), 7.20-7.80(\mathrm{~m}, 8 \mathrm{H}){ }^{13} \mathrm{C} \mathrm{NMR}\left(\mathrm{CDCl}_{3}\right): \delta 7.1,23.0,24.0,35.8$, $41.2,47.3,48.8,52.3,66.9,70.0,119.9,124.0,126.2,127.3,141.5$, 
144.4, 155.0, 171.8, 172.1; ES MS: $m / z 481.4[\mathrm{M}+\mathrm{H}]^{+}$, Anal. calcd. for $\mathrm{C}_{28} \mathrm{H}_{36} \mathrm{~N}_{2} \mathrm{O}_{5}: \mathrm{C}, 69.98 ; \mathrm{H}, 7.55 ; \mathrm{N}, 5.83$. Found: $\mathrm{C}, 69.90 ; \mathrm{H}, 7.51 ; \mathrm{N}$, $5.84 \%$.

Fmoc-Ac $\mathbf{c}_{7} \mathbf{c}-\mathrm{Ac}_{5} \mathbf{c}-\mathrm{OMe}:{ }^{1} \mathrm{H}$ NMR $\left(\mathrm{CDCl}_{3}\right): \delta$ 1.51-2.00 (m, 20H), $4.11(\mathrm{~s}, 3 \mathrm{H}), 4.31(\mathrm{t}, 2 \mathrm{H}), 4.50(\mathrm{~d}, 1 \mathrm{H}), 5.01(\mathrm{~s}, 1 \mathrm{H}), 6.50(\mathrm{~s}, 1 \mathrm{H}), 7.20$ $8.01(\mathrm{~m}, 8 \mathrm{H}) ;{ }^{[13]} \mathrm{C}$ NMR $\left(\mathrm{CDCl}_{3}\right): \delta 21.6,23.4,25.9,32.7,39.6,47.4$, 52.3, 63.9, 65.3, 66.9, 120.0, 124.1, 126.2, 127.3, 141.4, 144.7, 155.3, 170.1, 175.1; ES MS: $m / z$ 505.5 $[\mathrm{M}+\mathrm{H}]^{+}$, Anal. calcd. for $\mathrm{C}_{30} \mathrm{H}_{36} \mathrm{~N}_{2} \mathrm{O}_{5}$ : C, 71.41; H, 7.19; N, 5.55. Found: $\mathrm{C}, 71.32 ; \mathrm{H}, 7.16 ; \mathrm{N}$, $5.60 \%$.

\section{General Procedure for the Synthesis of $N^{\alpha}$-Protected Peptide Acids (6)}

To a stirred solution of Fmoc-amino acid chloride $(1 \mathrm{mmol})$ and HOBt-DCHA $(0.695 \mathrm{~g}, 2.2 \mathrm{mmol})$ in dry THF $(5 \mathrm{~mL})$, a TMS activated amino acid $(1.2 \mathrm{mmol})$ was added. Trimethylsilyl chloride (TMS-Cl, $1.2 \mathrm{mmol}$ ) and triethylamine (TEA, $2.5 \mathrm{mmol}$ ) were added to a stirred suspension of amino acid $(1 \mathrm{mmol})$ in DCM $(10 \mathrm{~mL})$ and refluxed for $2-4 \mathrm{~h}$. The completion of the reaction was monitored by TLC. The reaction mixture was concentrated to remove THF, and the residue was dissolved in sodium carbonate solution $(10 \%, 15 \mathrm{~mL})$. The aqueous phase was washed with ether $(2 \times 10 \mathrm{~mL})$ and acidified using dil. $\mathrm{HCl}$. The compound was extracted in ethyl acetate $(2 \times 10 \mathrm{~mL})$. The organic phase was washed with water $(2 \times 10 \mathrm{~mL})$ and brine $(10 \mathrm{~mL})$ and dried over anhydrous sodium sulphate. It was concentrated under vacuum to get the desired compound as a white solid in good yield.

\section{Data}

Fmoc-(D)Phg-Phe-OH: ${ }^{1} \mathrm{H}$ NMR (DMSO): $\delta 2.90$ (d, 2H), 3.70 (m, 2H), $4.25(\mathrm{~m}, 3 \mathrm{H}), 4.90(\mathrm{~d}, 1 \mathrm{H}), 5.31(\mathrm{~d}, 1 \mathrm{H}), 7.10-7.50(\mathrm{~m}, 14 \mathrm{H}), 7.55(\mathrm{~d}, 2 \mathrm{H})$, $7.80(\mathrm{~d}, 2 \mathrm{H}), 12.01(\mathrm{br}, 1 \mathrm{H}) ;{ }^{13} \mathrm{C}$ NMR (DMSO): $\delta 36.6,47.4,49.4,66.4$, 74.9, 120.0, 124.0, 125.0, 126.3, 126.4, 126.8, 127.3, 128.4, 129.6, 130.9, 133.6, 140.7, 141.5, 144.5, 155.3, 166.5, 172.7; ES MS: $m / z 521.3$ $[\mathrm{M}+\mathrm{H}]^{+}$, Anal. calcd. for $\mathrm{C}_{32} \mathrm{H}_{28} \mathrm{~N}_{2} \mathrm{O}_{5}: \mathrm{C}, 73.83 ; \mathrm{H}, 5.42 ; \mathrm{N}, 5.38$. Found: C, 73.79; H, 5.43; N, 5.40\%.

Fmoc-Tyr(Bn)-Pro-OH: ${ }^{1} \mathrm{H}$ NMR (DMSO): $\delta 2.12$ (m, 4H), 3.00 (d, $2 \mathrm{H}), 3.51(\mathrm{~m}, 2 \mathrm{H}), 3.82(\mathrm{t}, 1 \mathrm{H}), 4.20(\mathrm{~d}, 2 \mathrm{H}), 4.40(\mathrm{t}, 1 \mathrm{H}), 5.80(\mathrm{br}, 1 \mathrm{H})$, $7.20-7.90(\mathrm{~m}, 17 \mathrm{H}), 12.5(\mathrm{br}, 1 \mathrm{H}) ;{ }^{13} \mathrm{C}$ NMR (DMSO): $\delta 20.6,29.1,36.7$, 47.4, 52.0, 58.4, 60.1, 69.4, 74.4, 118.9, 120.0, 124.2, 127.0, 127.2, 127.6, 128.2, 129.9, 130.6, 136.8, 141.5, 144.7, 153.4, 157.5, 163.6, 175.4; ES MS: 
$m / z 577.4[\mathrm{M}+\mathrm{H}]^{+}$, Anal. calcd. for $\mathrm{C}_{35} \mathrm{H}_{32} \mathrm{~N}_{2} \mathrm{O}_{6}: \mathrm{C}, 72.90 ; \mathrm{H}, 5.59$; N, 4.86. Found: C, 72.82; H, 5.60; N, 4.88\%.

Fmoc-Asp(OBn)-Ala-OH: ${ }^{1} \mathrm{H}$ NMR (DMSO): $\delta 1.21(\mathrm{~d}, 3 \mathrm{H}), 2.60$ $(\mathrm{d}, 2 \mathrm{H}), 3.61-3.72(\mathrm{~m}, 2 \mathrm{H}), 4.20(\mathrm{t}, 1 \mathrm{H}), 4.31(\mathrm{~m}, 2 \mathrm{H}), 4.80(\mathrm{~s}, 2 \mathrm{H})$, $5.30(\mathrm{~d}, 1 \mathrm{H}), 5.70(\mathrm{br}, 1 \mathrm{H}), 7.10-7.52(\mathrm{~m}, 9 \mathrm{H}), 7.61(\mathrm{~d}, 2 \mathrm{H}), 7.80(\mathrm{~d}$, 2H), 8.00 (d, 1H), 12.4 (br, 1H); ${ }^{13} \mathrm{C}$ NMR (DMSO): $\delta$ 17.6, 39.3, 44.9, $47.2,54.8,63.8,66.5,120.0,124.0,126.2,127.2,128.1,128.6,141.5$, 142.8, 144.7, 155.4, 169.9, 171.0, 175.9; ES MS: $m / z 517.4[\mathrm{M}+\mathrm{H}]^{+}$, Anal. calcd. for $\mathrm{C}_{29} \mathrm{H}_{28} \mathrm{~N}_{2} \mathrm{O}_{7}$ : C, 67.43; H, 5.46; N, 5.42. Found: C, 67.40; H, 5.47; N, 5.45\%.

Fmoc-Ser(OBn)-Val-OH: ${ }^{1} \mathrm{H}$ NMR (DMSO): $\delta 0.91(\mathrm{t}, 6 \mathrm{H}), 1.81(\mathrm{~m}$, $1 \mathrm{H}), 3.40-3.72(\mathrm{~m}, 4 \mathrm{H}), 4.10(\mathrm{t}, 1 \mathrm{H}), 4.32(\mathrm{~d}, 2 \mathrm{H}), 4.91(\mathrm{~s}, 2 \mathrm{H}), 5.20(\mathrm{~d}$, 1H), 5.83 (br, 1H), 7.18-7.54 (m, 9H), 7.62 (d, 2H), 7.85 (d, 2H), 8.00 $(\mathrm{d}, 1 \mathrm{H}), 12.6(\mathrm{br}, 1 \mathrm{H}) ;{ }^{13} \mathrm{C}$ NMR (DMSO): $\delta 18.9,30.2,47.4,52.3$, $58.1,66.5,69.7,73.0,120.0,124.0,126.5,127.2,127.9,128.0,128.3$, $137.6,141.5,144.7,155.0,170.9,128.3,137.6,141.5,144.7,155.0$, 170.9, 174.8; ES MS: $m / z 517.5[\mathrm{M}+\mathrm{H}]^{+}$, Anal. calcd. for $\mathrm{C}_{30} \mathrm{H}_{32} \mathrm{~N}_{2} \mathrm{O}_{6}$ : C, 69.75; H, 6.24; N, 5.42. Found: C, 69.62; H, 6.21; N, $5.39 \%$.

\section{Synthesis of Fmoc-Val-Pro-Gly-Val-Gly-OBzl}

Fmoc-Val-Gly-Obn (I)

To H-Gly-OBn $(1.65 \mathrm{~g}, 10 \mathrm{mmol})$ in THF $(10 \mathrm{~mL})$, a mixture of FmocVal-Cl $(3.6 \mathrm{~g}, 10 \mathrm{mmol})$ and HOBt.DCHA $(3.5 \mathrm{~g}, 11 \mathrm{mmol})$ in THF $(20 \mathrm{~mL})$ was added and stirred untill the completion of reaction. The workup of the reaction mixture followed by column purification gave the peptide (I) as a white crystalline solid $(4.3 \mathrm{~g}, 90 \%) ; \mathrm{mp} 182-184{ }^{\circ} \mathrm{C}$; $[\alpha]^{25}{ }_{\mathrm{D}}+32.2^{\circ}\left(c 1.0, \mathrm{CHCl}_{3}\right) ; \mathrm{R}_{\mathrm{f}} \mathrm{A}, 0.51 ; \mathrm{R}_{\mathrm{f}} \mathrm{B}, 0.65 ;{ }^{1} \mathrm{H}$ NMR $\left(\mathrm{CDCl}_{3}\right)$ : $\delta$ 0.90-1.01 (d, $J=6.4 \mathrm{~Hz}, 6 \mathrm{H}), 1.29(\mathrm{~m}, 1 \mathrm{H}), 3.81(\mathrm{~m}, 1 \mathrm{H}), 4.20-4.40$ $(\mathrm{m}, 4 \mathrm{H}), 5.00(\mathrm{~s}, 2 \mathrm{H}), 6.70(\mathrm{~m}, 1 \mathrm{H}), 7.20-7.43(\mathrm{~m}, 9 \mathrm{H}), 7.58(\mathrm{~d}$, $J=6.9 \mathrm{~Hz}, 2 \mathrm{H}), 7.76(\mathrm{~d}, J=6.8 \mathrm{~Hz}, 2 \mathrm{H}) ;{ }^{13} \mathrm{C} \mathrm{NMR}\left(\mathrm{CDCl}_{3}\right): \delta 18.6$, $29.1,40.4,47.3,55.1,67.4,69.5,119.9,124.1,126.4,126.8,127.2$, 127.4, 128.7, 133.5, 141.5, 143.4, 155.2, 169.4, 172.4; ES MS: $m / z 509.3$ $[\mathrm{M}+\mathrm{Na}]^{+}$, Anal. calcd. for $\mathrm{C}_{29} \mathrm{H}_{30} \mathrm{~N}_{2} \mathrm{O}_{5}: \mathrm{C}, 71.59 ; \mathrm{H}, 6.21 ; \mathrm{N}, 5.76$, Found: C, 71.55; H, 6.19; N, 5.73\%.

\section{Fmoc-Gly-Val-Gly-OBn (II)}

Fmoc-Gly-Cl $(2.7 \mathrm{~g}, 8.8 \mathrm{mmol})$ and HOBt.DCHA $(3.1 \mathrm{~g}, 9.7 \mathrm{mmol})$ in THF $(20 \mathrm{~mL})$, were added to a solution of free peptide H-Val-Gly-OBn 
( $2.3 \mathrm{~g}, 8.8 \mathrm{mmol})$, obtained by the deprotection of I ( $4.3 \mathrm{~g}, 9 \mathrm{mmol})$ using TAEA $^{[4]}(45 \mathrm{~mL})$ in THF $(10 \mathrm{~mL})$. Workup of the reaction mixture followed by column purification gave the peptide (II) as a white crystalline solid $(4.4 \mathrm{~g}, 88 \%) ; \mathrm{mp} 176-179^{\circ} \mathrm{C} ;[\alpha]^{[25]} \mathrm{D}+30.4^{\circ}\left(\mathrm{c} 1.0, \mathrm{CHCl}_{3}\right) ;{ }^{1} \mathrm{H}$ NMR $\left(\mathrm{CDCl}_{3}\right): \delta 0.90-1.01(\mathrm{~d}, J=6.3 \mathrm{~Hz}, 6 \mathrm{H}), 1.30(\mathrm{~m}, 1 \mathrm{H}), 3.67(\mathrm{~m}$, $4 \mathrm{H}), 3.93(\mathrm{~m}, 1 \mathrm{H}), 4.20-4.45(\mathrm{~m}, 5 \mathrm{H}), 5.01(\mathrm{~s}, 2 \mathrm{H}), 6.20(\mathrm{~d}, 1 \mathrm{H}), 7.20$ $7.81(\mathrm{~m}, 13 \mathrm{H}),{ }^{13} \mathrm{C} \mathrm{NMR}\left(\mathrm{CDCl}_{3}\right): \delta 18.6,29.1,40.4,46.2,47.1,57.5$, 67.4, 69.5, 119.9, 124.1, 126.2, 126.9, 127.2, 127.4, 128.7, 133.2, 141.5, 143.4, 157.1, 165.4, 169.4, 173.4; ES MS: $m / z 566.2[\mathrm{M}+\mathrm{Na}]^{+}$, Anal. calcd. for $\mathrm{C}_{31} \mathrm{H}_{33} \mathrm{~N}_{3} \mathrm{O}_{6}: \mathrm{C}, 68.49 ; \mathrm{H}, 6.12 ; \mathrm{N}, 7.73$, Found: $\mathrm{C}, 68.55 ; \mathrm{H}$, $6.19 ; \mathrm{N}, 5.79 \%$.

Fmoc-Pro-Gly-Val-Gly-OBn (III)

Fmoc-Pro-Cl (2.6 g, $8.0 \mathrm{mmol})$ and HOBt-DCHA (2.78 g, $8.8 \mathrm{mmol})$ in THF $(20 \mathrm{~mL})$ were added to a solution of the free peptide H-Gly-ValGly-OBn $(2.2 \mathrm{~g}, 8.0 \mathrm{mmol})$, obtained by the deprotection of the peptide (II) $(4.4 \mathrm{~g}, 8.1 \mathrm{mmol})$ using tris(2-aminoethyl)amine (TAEA) $(40 \mathrm{~mL})$ in THF $(10 \mathrm{~mL})$. Workup of the reaction mixture followed by column purification gave the title product (III) as a crystalline solid $(4.6 \mathrm{~g}, 78 \%) \mathrm{mp}$ $158-60^{\circ} \mathrm{C} ;[\alpha]^{25}{ }_{\mathrm{D}}+24.2^{\circ}\left(\mathrm{c} 1.0, \mathrm{CHCl}_{3}\right) ;{ }^{1} \mathrm{H}$ NMR $\left(\mathrm{CDCl}_{3}\right): \delta 0.90-1.01$ $(\mathrm{d}, J=6.7 \mathrm{~Hz}, 6 \mathrm{H}), 1.29(\mathrm{~m}, 1 \mathrm{H}), 1.73-2.24(\mathrm{~m}, 6 \mathrm{H}), 3.67(\mathrm{~m}, 4 \mathrm{H})$, $3.93(\mathrm{~m}, 1 \mathrm{H}), 4.11(\mathrm{t}, 1 \mathrm{H}), 4.20-4.45(\mathrm{~m}, 5 \mathrm{H}), 5.00(\mathrm{~s}, 2 \mathrm{H}), 5.10(\mathrm{~d}$, $1 \mathrm{H}), 6.20(\mathrm{~d}, 1 \mathrm{H}), 7.20-7.81(\mathrm{~m}, 13 \mathrm{H}) ;{ }^{13} \mathrm{C} \mathrm{NMR}\left(\mathrm{CDCl}_{3}\right): \delta 18.6,26.8$, 28.4, 29.4, 40.2, 43.6, 46.9, 47.3, 57.5, 59.4, 67.4, 69.6, 119.9, 124.1, 126.4, 127.0, 127.2, 127.4, 128.9, 133.3, 141.5, 143.7, 165.5, 167.8, 167.9, 169.4, 170.2; ES MS: $m / z 663.7[\mathrm{M}+\mathrm{Na}]^{+}$, Anal. calcd. for $\mathrm{C}_{36} \mathrm{H}_{40} \mathrm{~N}_{4} \mathrm{O}_{7}: \mathrm{C}$, 67.48; H, 6.29; N, 8.74, Found: C, 67.20; H, 6.29; N, 8.73\%.

\section{Fmoc-Val-Pro-Gly-Val-Gly-OBn (IV)}

Fmoc-Val-Cl (2.3 g, $7.0 \mathrm{mmol})$ and HOBt.DCHA (2.43 g, $7.7 \mathrm{mmol})$ in THF $(20 \mathrm{~mL})$ were added to a solution of the free peptide H-Pro-GlyVal-Gly-OBn $(2.91 \mathrm{~g}, 7.0 \mathrm{mmol})$, obtained by the deprotection of the peptide (III) $(4.6 \mathrm{~g}, 7.1 \mathrm{mmol})$ using TAEA $(35 \mathrm{~mL})$ in THF $(10 \mathrm{~mL})$. Workup of the reaction mixture followed by column purification gave the title product (IV) as a crystalline solid $(4.6 \mathrm{~g}, 67 \%) ; \mathrm{mp} 98-100^{\circ} \mathrm{C}$; $[\alpha]^{25} \mathrm{D}^{-18.7^{\circ}}\left(c 1.0, \mathrm{CHCl}_{3}\right) ;{ }^{1} \mathrm{H}$ NMR $\left(\mathrm{CDCl}_{3}\right): \delta 0.89-1.10(\mathrm{~m}, 12 \mathrm{H})$, $1.28(\mathrm{~m}, 2 \mathrm{H}), 1.73-2.24(\mathrm{~m}, 6 \mathrm{H}), 3.65(\mathrm{~d}, 1 \mathrm{H}), 3.95(\mathrm{~m}, 1 \mathrm{H}), 4.02(\mathrm{~d}$, $2 \mathrm{H}), 4.13(\mathrm{~d}, 2 \mathrm{H}), 4.18(\mathrm{~m}, 3 \mathrm{H}), 4.20-4.40(\mathrm{~m}, 2 \mathrm{H}), 4.45(\mathrm{t}, 1 \mathrm{H}), 5.01$ $(\mathrm{s}, 2 \mathrm{H}), 5.10(\mathrm{~m}, 2 \mathrm{H}), 6.01(\mathrm{~m}, 1 \mathrm{H}), 7.20-7.84(\mathrm{~m}, 13 \mathrm{H}) ;{ }^{13} \mathrm{C} \mathrm{NMR}$ $\left(\mathrm{CDCl}_{3}\right): \delta 18.6,18.8,27.0,27.3,30.1,30.2,40.1,42.4,47.1,48.4,54.6$, 
55.9, 58.6, 67.4, 69.4, 119.9, 124.1, 126.2, 127.0, 127.3, 127.5, 128.7, 133.5, 141.5, 143.4, 155.2, 165.4, 167.8, 167.9, 168.4, 170.4; ES MS: $m / z 763.7$ $[\mathrm{M}+\mathrm{Na}]^{+}$, Anal. calcd. for $\mathrm{C}_{41} \mathrm{H}_{49} \mathrm{~N}_{5} \mathrm{O}_{8}: \mathrm{C}, 66.56 ; \mathrm{H}, 6.68 ; \mathrm{N}, 9.47$, Found: C, 66.47; H, 6.57; N, 9.40\%.

\section{Synthesis of $\beta$-Casomorphin (H-Tyr-Pro-Phe-Pro-Gly-OH)}

The peptide was synthesized using manual Fmoc-chemistry with a solid-phase synthesis strategy on Wang resin (substitution $0.6 \mathrm{mmol} / \mathrm{g}$ ) employing Fmoc-Tyr(OBzl)-COCl $(0.92 \mathrm{mg}, 1.8 \mathrm{mmol}, 3 \mathrm{eq})$, FmocPro-COCl $(0.64 \mathrm{mg}, \quad 1.8 \mathrm{mmol}, 3$ eq), Fmoc-Phe-COCl $(0.72 \mathrm{mg}$, $1.8 \mathrm{mmol}, 3 \mathrm{eq})$, Fmoc-Pro-COCl $(0.64 \mathrm{mg}, 1.8 \mathrm{mmol}, 3 \mathrm{eq})$, and HOBt.DCHA $(0.63 \mathrm{mg}, 1.98 \mathrm{mmol}, 3.1 \mathrm{eq})$ in $\mathrm{DMF}-\mathrm{CH}_{2} \mathrm{Cl}_{2}(10 \mathrm{~mL}$, 1:1). The synthesis was carried out using $1 \mathrm{~g}$ of Fmoc-Gly-Wang resin. A single coupling for $30 \mathrm{~min}$ was sufficient for the completion of each coupling. The DCHA. $\mathrm{HCl}$ formed as a white precipitate was removed by washings using $\mathrm{CHCl}_{3}-\mathrm{CH}_{2} \mathrm{Cl}_{2}$ (1:1). After deprotection of Fmoc group using $20 \%$ pipyridine in $\mathrm{DMF}$, the peptide was cleaved using trifluroacetic acid (TFA) $(10 \mathrm{~mL}) / \mathrm{H}_{2} \mathrm{O}(0.5 \mathrm{~mL}) /$ thioanisole $(0.5 \mathrm{~mL}) /$ phenol $(0.75 \mathrm{~g})$ mixture at room temperature for $1 \mathrm{~h}$. The crude peptide was subjected to catalytic hydrogenation using $10 \% \mathrm{Pd} / \mathrm{C}(50 \mathrm{mg}) /$ methanol $(10 \mathrm{~mL}) /$ hydrogen gas to remove the benzyl group and was purified by RP-HPLC to obtain the final peptide $(275 \mathrm{mg}$, $79 \%) . \mathrm{Mp} 151-153{ }^{\circ} \mathrm{C} ;[\alpha]^{[25]} \mathrm{D}^{-48.5^{\circ}}$ (c 0.5, DMF); ${ }^{1} \mathrm{H}$ NMR $\left(\mathrm{CDCl}_{3}\right)$ : $\delta 1.10-2.10(\mathrm{~m}, 14 \mathrm{H}), 2.31(\mathrm{t}, 2 \mathrm{H}), 2.90(\mathrm{t}, 2 \mathrm{H}), 3.11(\mathrm{t}, 1 \mathrm{H}), 5.20(\mathrm{br}$, $1 \mathrm{H}), 5.40$ (br, 1H), 5.71 (br, $1 \mathrm{H}), 7.20-8.10(\mathrm{~m}, 9 \mathrm{H}), 12.0-13.0(1 \mathrm{H}$, $\mathrm{COOH}) ;{ }^{13} \mathrm{C}$ NMR $\left(\mathrm{CDCl}_{3}\right): \delta 27.3,29.0,33.9,35.4,36.1,50.6,51.3$, $51.8,54.3,58.1,58.8,127.1,128.9,129.4,129.5,130.1,130.2,140.1$, 155.4, 166.0, 168.6, 169.4, 170.3, 177.2; ES MS: $m / z 602.65[\mathrm{M}+\mathrm{Na}]^{+}$, , Anal. calcd. for $\mathrm{C}_{30} \mathrm{H}_{37} \mathrm{~N}_{5} \mathrm{O}_{7}$ : C, 62.16; H, 6.43; N, 12.08, Found: $\mathrm{C}$, $62.12 ; \mathrm{H}, 6.40 ; \mathrm{N}, 12.00 \%$; R, 8.45 [(Waters $\mathrm{C}-18$ deltapak column $(3.9 \times 300 \mathrm{~mm}, 15 \mu)$; using eluent acetonitrile $0.1 \%$ trifluoroacetic acid (TFA) and water (65:35; isocratic, flow rater $1 \mathrm{~mL} / \mathrm{min}$, monitoring at $220 \mathrm{~nm})$.

\section{ACKNOWLEDGMENTS}

We are grateful to the Department of Science and Technology, Government of India, for financial support. We thank Professor Fred Naider, City of University of New York (CUNY), New York, for useful discussions and S. I. F, and M. B. U. of Indian Institute of Science (IISc), Bangalore, for providing NMR and mass facilities. 


\section{REFERENCES}

1. Carpino, L. A.; Cohen, B. J.; Stephens Jr., K. E.; Sadat-Aalaee, S. Y.; Tien, J.-H.; Langridge, D. C. ((9-Fluorenylmethy1)oxy)carbonyl (Fmoc) amino acid chlorides: Synthesis, characterization, and application to the rapid synthesis of short peptide segments. J. Org. Chem. 1986, 51, 3732.

2. Carpino, L. A. Methyl ester nonequivalence in the ${ }^{1} \mathrm{H}$ NMR spectra of diastereomeric dipeptide esters incorporating $\mathrm{N}$-terminal a-phenylglycine units. J. Org. Chem. 1988, 53(4), 875.

3. Beyermann, M.; Bienert, M.; Niedrich, H.; Carpino, L. A.; Sadat-Aalaee, D. Rapid continuous peptide synthesis via Fmoc amino acid chloride coupling and 4-(aminomethyl)piperidine deblocking. J. Org. Chem. 1990, 55, 721.

4. Beyermann, M.; Carpino, L. A.; Sadat-Aalaee, D. Tris(2-aminoethy1)amine as a substitute for 4-(aminomethy1)piperidine in the Fmoc/polyamine approach to rapid peptide synthesis. J. Org. Chem. 1990, 55, 1673.

5. Beyermann, M.; Carpino, L. A.; Chao, H. G.; Bienert, M. (9-Fluorenylmethyl)oxy carbonyl amino acid chlorides in solid-phase peptide synthesis. J. Org. Chem. 1991, 56, 2635.

6. Perlow, D. S.; Erb, J. M.; Gould, N. P.; Tung, R. D.; Freidinger, R. M.; Williams, P. D.; Veber, D. F. Use of N-Fmoc amino acid chlorides and activated 2-(fluorenylmethoxy)-5(4H)-oxazolones in solid-phase peptide synthesis: Efficient syntheses of highly $\mathrm{N}$-alkylated cyclic hexapeptide oxytocin antagonists related to L-365, 209. J. Org. Chem. 1992, 57, 4394.

7. Williams, P. D.; Bock, M. G.; Tung, R. D.; Garsky, V. M.; Perlow, D. S.; Erb, J. M.; Lundell, G. F.; Gould, N. P.; Whitfer, W. L.; Hoffman, J. B.; Kaufman, M. J.; Clineschmidt, B.U.; Pettibone, D. T.; Freidinger, R. M.; Veber, D. F. Development of a novel class of cyclic hexapeptide oxytocin antagonists based on a natural product. J. Med.Chem. 1992, 35, 3905.

8. Sivanandaiah, K. M.; Sureshbabu, V. V.; Shankaramma, S. C. Acid chloride/KOBt as a coupling agent in peptide synthesis. Indian J. Chem. 1992, $31 B, 379$.

9. Sivanandaiah, K. M.; Sureshbabu, V. V.; Shankaramma, S. C. Synthesis of peptides mediated by KOBt. Int. J. Peptide Protein Res. 1994, 44, 24.

10. Sureshbabu, V. V.; Gopi, H. N. Synthesis of peptides mediated by potassium salt of 1-hydroxy-7-azabenzotriazole. Indian J. Chem. 1998, 37B, 394.

11. Sureshbabu, V. V.; Gopi, H. N. Synthesis of $\left[\mathrm{Leu}^{5}\right]$ enkephalin using Fmoc-amino acid chlorides/KOBt. Indian J. Chem. 1998, 75, 511.

12. Sureshbabu, V. V.; Tantry, S. J. 1-(t-Butyldimethylsilyloxy)benzotriazole (TBDMS-OBt): A new and novel reagent for the synthesis of peptides. Lett. Peptide Sci. 2002, 9, 35.

13. Sureshbabu, V. V.; Tantry, S. J.; Mathad, R. I. Synthesis of $\beta$-casomorphin employing Fmoc-amino acid chlorides and t-butyldimethylsilyloxybenzotriazole. Indian J. Chem. 2003, 42 B, 2104.

14. Sureshbabu, V. V.; Gopi, H. N. Synthesis of peptides employing Fmoc-amino acid chlorides and commercial zinc dust. Tetrahedron Lett. 1998, 39, 9769. 
15. Sureshbabu, V. V.; Gopi, H. N.; Ananda, K. Zinc promoted simple synthesis of Z-amino acids under neutral conditions. Protein and Peptide Lett. 1999, 6(4), 233.

16. Urry, D. W.; Cunning, W. D.; Ohnishi, T. Studies on the conformation and interactions of Elastin proton magnetic resonance of the repeating pentapeptidet. Biochemistry 1974, 13, 609.

17. (a) Konig, W.; Geiger, R. Rasemization in peptide synthesis. Chem. Ber. 1970, 103, 2024; (b) Konig, W.; Geiger, R. A new method for synthesis of peptides: Activation of the carboxyl group with dicyclohexylcarbodiimide using 1-hydroxybenzotriazoles as additives. Chem. Ber. 1970, 103, 788.

18. Dourtoglou, V.; Ziegler, J.-C.; Gross, B. L'hexafluorophosphate de O-benzotriazolyl-N, N-tetramethyluronium: Un reactif de couplage peptidique nouveau et efficace. Tetrahedron Lett. 1978, 19, 1269.

19. Knorr, R.; Trzeuak, A.; Bannwarth, W.; Gillessen, D. New coupling reagents in peptide chemistry. Tetrahedron Lett. 1999, 30, 1927.

20. Carpino, L. A.; Han, G. Y. The 9-fluorenylmethoxycarbonyl function, a new base-sensitive amino-protecting group. J. Am. Chem. Soc. 1970, 92, 5748.

21. Katritzky, A. R.; Angrish, P.; Hur, D.; Suzuki, K. N-(Cbz- and Fmoc- $\alpha$-aminoacyl)benzotriazoles: Stable derivatives enabling peptide coupling of Tyr, Trp, Cys, Met, and Gln with free amino acids in aqueous media with complete retention ofchirality. Synthesis 2005, 3, 397.

22. Wilkening, R. R.; Stevens, E. S.; Bonora, G. M.; Toniolo, C. Conformational analysis of linear peptides, 4: Association properties of protected oligomers of $\alpha$-aminoisobutyric acid in chloroform. J. Am. Chem. Soc. 1983, 105, 2560.

23. Prasad, S.; Balaji R.; Balaram, P. Contrasting solution conformations of peptides containing $\alpha \alpha$-dialkylated residues with linear and cyclic side chains. Biopolymers 1995, 35, 11.

24. Bolin, R. D.; Sytwu, I. I.; Humiec, F.; Meinhoffer, J. Preparation of oligomer-free $N^{\alpha}$-Fmoc and $N^{\alpha}$-urethane amino acids. Int. J. Peptide Protein Res. 1989, 33, 353.

25. Wenschuh, H.; Beyermann, M.; E1-Faham, A.; Ghassemi, S.; Carpino, L. A.; Bienert, M. Peptide assembly in the absence of base via Fmoc-amino acid fluorides. J. Chem. Soc. Chem. Commun. 1995, 669.

26. Carpino, L. A.; Sadat-Aalaee, D.; Chao, H. G.; Deselms, R. H. ((9-Fluorenylmethyl)oxy)carbonyl (FMOC) amino acid fluorides: Convenient new peptide coupling reagents applicable to the FMOCl tert-butyl strategy for solution and solid-phase syntheses. J. Am. Chem. Soc. 1990, 112, 9651.

27. Carpino, L. A.; Mansour, E. S. M. E.; Sadat-Aalaee, D. tert-Butyloxycarbonyl and benzyloxycarbonyl amino acid fluorides: New, stable, rapid-acting acylating agents for peptide synthesis. J. Org. Chem. 1991, 56, 2611.

28. Paquet, A. Introduction of 9-fluorenylmethyloxycarbonyl, trichloroethoxycarbonyl, and benzyloxycarbonyl amine protecting groups into O-unprotected hydroxyamino acids using succinimidyl carbonates. Can. J. Chem. 1982, 60, 976. 